\title{
Les formats alternatifs aux anticorps
}

Fragments et nouvelles charpentes

$>$ Les anticorps sont désormais devenus d'une utilisation courante dans un large champ thérapeutique qui n'est plus restreint à la cancérologie et à l'inflammation. Cette explosion du domaine conduit à des besoins nouveaux qui peuvent être mieux remplis par des molécules inspirées mais différentes des anticorps classiques. En particulier, la molécule anticorps a de multiples fonctions qui ne sont pas toujours nécessaires, comme sa capacité à recruter les cellules du système immunitaire, à se lier de façon bivalente à sa cible ou à présenter une demi-vie plasmatique élevée. En revanche, dans la grande majorité des applications, sa remarquable capacité à reconnaître spécifiquement sa cible moléculaire et surtout sa diversité de reconnaissance doivent être conservées. De plus, les anticorps sont des molécules de très haut poids moléculaire, coûteuses à produire et qui présentent des propriétés physicochimiques limitées ne permettant pas leur utilisation dans des milieux agressifs. Finalement, dans certaines applications thérapeutiques, la grande taille de la molécule (environ $150 \mathrm{kDa}$ ) peut également limiter sa diffusion dans les tissus et empêcher la reconnaissance de certaines structures moléculaires peu accessibles. Pour répondre à ces limitations, de nombreux formats alternatifs aux anticorps entiers ont été développés au cours de ces vingt dernières années. Les applications couvrent les domaines de la biotechnologie, du diagnostic in vitro et in vivo et de la thérapie. Deux grandes familles de molécules permettent de couvrir ce champ et seront présentées dans cette mini-revue. Une première famille s'appuie sur la diversité naturelle des anticorps mais en en réduisant la taille, comme les fragments d'anticorps classiques (Fab, scFv) ou ceux provenant des camélidés ou des requins (VHH, V-NAR). La deuxième famille a été développée en partant

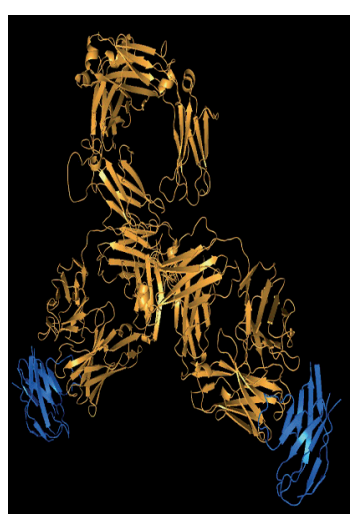

${ }^{1}$ Affilogic, Nantes, France. ${ }^{2}$ IRCM, Institut de Recherche en Cancérologie de Montpellier, Inserm U1194, Université de Montpellier, Institut régional du Cancer de Montpellier, Montpellier, France. olivier@affilogic.com pierre.martineau@inserm.fr

des propriétés finales désirées et notamment la stabilité en milieu extrême et la productivité en système simple et économique de production comme l'utilisation de bactéries et en y greffant des propriétés de liaison comparables aux anticorps par des méthodes d'évolution moléculaire dirigée in vitro. Cette mini-revue se concentrera sur les molécules les plus avancées, mais le domaine est en très forte et rapide expansion. II faut noter que beaucoup de ces molécules, voire ces approches, sont couvertes par des brevets et sont souvent développées dans le cadre de jeunes sociétés innovantes dont certaines ont déjà été rachetées par de grands groupes de la pharmacie. <

\section{Les fragments d'anticorps}

La structure des anticorps est connue depuis les années 1960 grâce aux travaux de Rodney R. Porter pour lesquels il obtiendra le prix Nobel de physiologie ou médecine en 1972. Porter a notamment démontré qu'il est possible de générer à partir d'un anticorps entier un fragment, dit Fab pour fragment antigen-binding, d'une taille trois fois plus petite mais présentant les mêmes propriétés de liaison à l'antigène que la molécule d'origine. Par la suite et à l'aide des méthodes de génie génétique, des molécules de plus en plus petites fondées sur la molécule anticorps ont été générées. Tout d'abord, il a été montré que l'on pouvait ne conserver que la moitié d'un Fab et produire uniquement 
un fragment constitué des deux domaines $\mathrm{N}$-terminaux des chaînes lourdes et légères, c'est-à-dire la partie dite Fv (fragment variable) des anticorps. Pour augmenter la stabilité de cette construction il a ultérieurement été proposé de la produire sous la forme dite simplechaîne, le scFv, pour single-chain Fv, associant les deux domaines variables en une protéine unique à l'aide d'un peptide flexible d'une quinzaine d'acides aminés. Finalement, il a été montré que certains Fv pouvaient même être encore divisés en deux et produire des domaines de très petite taille fondés sur l'unique domaine variable de la chaîne lourde mais présentant encore une forte capacité de liaison à l'antigène : ces mini-anticorps ont été appelés sdAb pour single-domain antibody et représentent le plus petit domaine des anticorps de mammifère présentant une activité de liaison [1] (Figure 1).

Ces sdAb sont toutefois restés peu utilisés car ils présentaient des propriétés de stabilité et surtout de solubilité défavorables. La découverte fortuite en 1993 d'anticorps naturellement dépourvus de chaîne légère chez le dromadaire a rapidement changé la donne en donnant accès à des $s d A b$ naturels présentant, au contraire, des propriétés physicochimiques remarquablement supérieures à celles des anticorps entiers [2]. Ces anticorps dit HcAb pour heavy chain-only antibody se lient à leur antigène uniquement par leur domaine $\mathrm{VH}$, appelé dans ce cas VHH. Ce domaine VHH est donc le domaine anticorps le plus petit produit de façon naturelle et est souvent appelé nanobody par référence à sa petite taille. II a rapidement été mis en évidence que ces anticorps $\mathrm{HcAb}$ étaient présents chez tous les camélidés dont les chameaux, les lamas et les vigognes. Contrairement aux sdAb issus des anticorps humains, ces VHH peuvent être produits de façon efficace par les bactéries et les levures, ils présentent une stabilité et une solubilité importantes et des propriétés de réversibilité de leur dénaturation par la chaleur proche de $100 \%$ [3], ce qui en fait des outils biotechnologiques originaux. Finalement, ces VHH ont des séquences d'acides aminés très fortement similaires à celles des anticorps humains ce qui en fait des molécules utilisables chez l'homme, minimisant ainsi les risques de l'induction d'une réponse immunitaire. Les anticorps simple chaîne ne sont pas restreints aux camélidés et ont également été décrits en 1995 chez certains poissons cartilagineux, comme le requin ou la raie, et sont appelés dans ce cas IgNAR (new antigen receptor) et V-NAR pour leur domaine variable. Bien que partageant avec les VHH leur nature monochaîne, les V-NAR sont d'une origine différente et représentent vraisemblablement une troisième branche, en plus de celles des récepteurs des lymphocytes $T$ et des anticorps, dérivant d'une forme ancestrale commune des récepteurs du système immunitaire [4].

L'ensemble de ces molécules permet la génération de protéines se liant à une cible, ces protéines ayant une très large gamme de masses moléculaires. On peut ainsi couvrir toute la gamme de taille entre des anticorps entiers de $150 \mathrm{kDa}$ et des VHH de $12 \mathrm{kDa}$ en passant par des molécules de 50 ou $25 \mathrm{kDa}$. Ce choix permet d'optimiser les propriétés de la molécule en fonction des objectifs recherchés. Par exemple, les molécules de masse inférieure à environ $50 \mathrm{kDa}$ sont rapidement éliminées dans les urines et pourront également diffuser plus profondément et rapidement dans des tissus et dans les tumeurs. Dans le cas des VHH, ces propriétés ont été exploitées pour développer des méthodes d'imagerie in vivo permettant une détection plus efficace des tumeurs que les anticorps entiers [5]. Une application particulière mais très intéressante des formats du type VHH et V-NAR est la génération de molécules à large spectre reconnaissant des parties conservées des pathogènes, non accessible à des molécules de la taille des anticorps et invisibles à la détection par le système immunitaire humain comme dans le cas des virus $[1,6]$ ou des parasites [7].

Ces molécules, Fab, Fv, scFv, VHH et V-NAR, peuvent être produites efficacement chez la bactérie ou la levure car elles ne nécessitent pas la machinerie de modification post-traductionelle présente dans les cellules de mammifère et indispensable pour la production d'anticorps entiers actifs. Les formats simple chaîne (scFv, VHH, V-NAR) sont également facilement modulables et peuvent servir de brique élémentaire pour la construction de molécules complexes à la façon d'un jeu de Lego ${ }^{\circledR}$. Cette dernière propriété a largement été utilisée pour la fabrication de molécules d'intérêt thérapeutique et pour construire des protéines multifonctionnelles et à durée de vie plasmatique élevée.

Finalement, ces molécules de petite taille conservent intact le domaine de reconnaissance des anticorps naturels. La connaissance accumulée sur l'interaction antigène-anticorps peut donc être directement exploitée pour sélectionner ou améliorer ces molécules. On sait par exemple qu'il est possible d'identifier des molécules contre virtuellement n'importe quel antigène et potentiellement contre la majeure partie, voire l'ensemble, de la surface d'une protéine cible $[8,9]$. Cette dernière propriété du système immunitaire, directement extrapolable aux fragments d'anticorps, est cruciale pour le développement de molécules présentant les propriétés désirées. Cela permet aussi d'utiliser cette connaissance pour faire évoluer ces molécules de façon rationnelle ou pour construire des banques naïves ou synthétiques [10].

\section{Les charpentes alternatives aux anticorps}

Une approche inverse consiste à s'intéresser à des molécules que la nature a déjà fait évoluer pour présenter les propriétés élémentaires recherchées (taille, stabilité, absence de fonctions effectrices) et auxquelles vont être ajoutées les fonctions supplémentaires désirées, généralement la capacité de reconnaissance et d'interaction avec une cible. Cette fonction, qui va guider la structure finale, peut être obtenue en l'espace de quelques semaines, voire quelques jours, grâce à des technologies aujourd'hui répandues d'ingénierie et d'évolution des protéines in vitro. Le concept qui sous- 


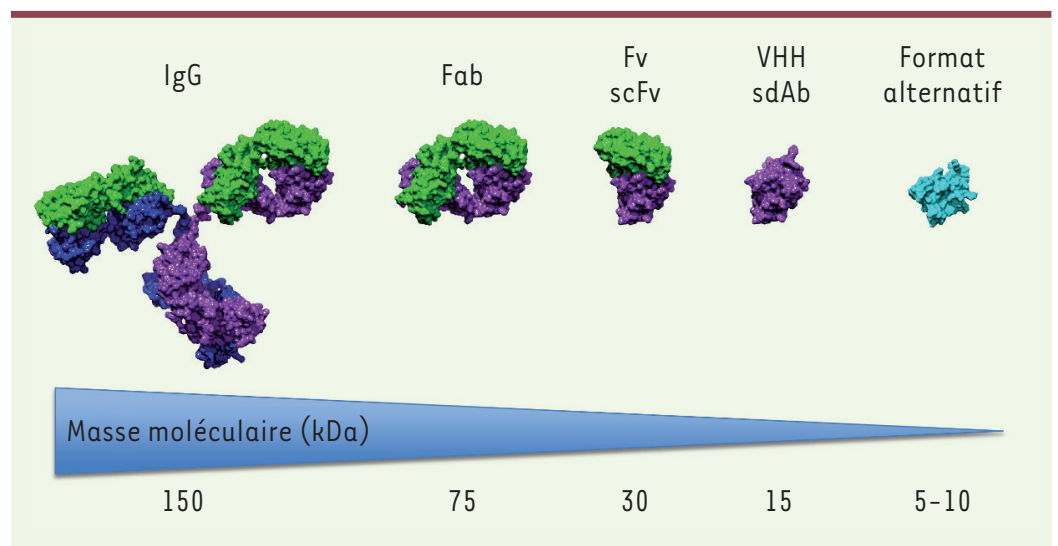

Figure 1. De l'anticorps aux nouvelles charpentes, un large choix de molécules et de tailles. d'une cible choisie sont disponibles, qu'ils sont simples à conjuguer sur des supports de purification et qu'ils sont suffisamment stables pour supporter les méthodes de régénération de ces colonnes, il devient possible de concevoir des systèmes alternatifs pour les anticorps ou d'autres protéines. Plusieurs charpentes ont été utilisées pour ces applications : les affibodies comme nouvelle génération de protéine $A$, les affilins et les nanofitines (programme de purification de vaccins DiViNe cofinancé par la commission européenne). Les performances sont démontrées dans un contexte industriel (affibody pour les anticorps) et pilote (affilogic pour différents vaccins). En particulier, tend cette approche est bien connu des ingénieurs des protéines: les éléments naturellement très stables sont également très tolérants aux mutations, insertions et délétions.

L'exploration de la biodiversité a permis de recenser des protéines présentant des caractéristiques intéressantes pour ces applications. C'est ainsi que sont apparues des familles de molécules aux noms aussi exotiques que affibodies, affilins, affimers, alphabodies, alpha-reps, anticalins, atrimers, avimers, centyrins, DARPins (pour «designed ankyrin repeat proteins »), fynomers, Kunitz domains, nanofitines (ou affitines), obodies, pronectins, ou repebodies [11-13]. Ces molécules sont fondées sur des éléments naturellement stables et de petite taille (moins de 100 acides aminés) qui servent de charpente pour la présentation de sites de liaison.

Pour générer ces nouveaux sites de liaison, deux approches principales sont utilisées: les domaines répétés ou la mutagénèse de domaines des protéines parentales. De façon générale, le principe d’identification de charpentes spécifiques pour une cible choisie repose sur un principe de sélection puis de criblage : des bibliothèques de variants de ces protéines sont générées par mutagénèse, puis les variants spécifiques d'une cible choisie sont sélectionnés en utilisant des technologies dites de présentation (display). Ces méthodes très puissantes permettent de cribler des bibliothèques d'une très grande diversité, pouvant aller jusqu'à $10^{13}$ variants [14] en quelques jours.

La fonction essentielle de ces charpentes rendues sélectives est de se lier à leur cible avec une cinétique qui, elle aussi, est sujette à ingénierie. De ce fait, elles ont longtemps été assimilées à des outils de capture ou de ciblage (vecteurs). Les applications principalement considérées étaient la séparation, l'imagerie ou, plus récemment, en tant que vecteur transportant des toxines vers les cellules d'intérêt, une alternative aux antibody-drug conjugates (ADC). Cependant, des résultats prometteurs permettent d'envisager également des applications thérapeutiques où la charpente est elle-même l'agent actif.

L'utilisation de la chromatographie d'affinité pour la purification de protéines biothérapeutiques est très largement dominée par la purification des anticorps monoclonaux (AcM) à l'aide de billes fonctionnalisées avec la protéine $A$, une protéine bactérienne liant avec une forte affinité les immunoglobulines $G(\operatorname{IgG})$. Dès lors que des ligands sélectifs la génération à façon de nanofitines dans le cadre de ce programme DiViNe a permis de montrer qu'une seule étape de chromatographie sélective peut remplacer jusqu'à 5 étapes traditionnelles, et que les colonnes ainsi générées sont recyclables plus de trente fois sans perte de performance.

L'imagerie est également un champ très intéressant pour des ligands de petite taille et favorables au radiomarquage [15]. La petite taille permet en effet une élimination rénale très rapide des vecteurs non associés à leur cible. La conséquence directe est un contraste élevé observable dans un temps très court, de l'ordre de l'heure chez le petit animal. Ce type d'approche rend I'utilisation de radioisotopes à courte demi-vie très attractive, réduisant ainsi les contraintes d'élimination des déchets et de radioprotection [16].

Ce profil d'élimination rapide est également attractif pour les approches du type immunotoxine. Un vecteur sélectif d'une tumeur et très rapidement éliminé s'il n'y est pas lié, présente, sur le papier, un gain certain en termes d'innocuité pour les cellules distantes. Ceci est d'autant plus vrai que les charpentes utilisées pour ce vecteur ne présentent pas d'activité de recyclage cellulaire via le récepteur néonatal FcRn. Leur interaction est donc très majoritairement limitée aux cellules portant l'antigène ciblé. Cependant, l'historique des anticorps et des $A D C$ suggère qu'une longue présence plasmatique permettrait une meilleure diffusion tumorale et serait un facteur déterminant de l'activité. L'avantage compétitif supposé des charpentes alternatives serait alors une rapidité supérieure de diffusion au sein des tumeurs, permettant d'atteindre une proportion élevée et spécifique des cellules en un seul passage. Cette hypothèse, modélisée par le Massachusetts Institute of Technology (MIT) $[17,18]$, est en cours de vérification et, déjà, les expériences de distribution intra-tumorale effectuées sont très convaincantes. 
Les usages de charpentes comme agents thérapeutiques actifs avec des données cliniques sont encore peu nombreux. Les DARPins et les anticalins sont parmi les molécules les plus avancées et pionnières dans les programmes de développement.

\section{Les applications cliniques}

Les molécules non conventionnelles sont encore fortement minoritaires en clinique. En effet, les succès des immunothérapies utilisant des anticorps entiers ont fortement incité les entreprises du médicament à développer en priorité des traitements utilisant les anticorps. De plus, ces petites molécules présentent des stabilités plasmatiques réduites et sont dépourvues des propriétés de recrutement des cellules immunitaires exprimant des récepteurs pour la région Fc des IgG (RFc gamma) dues à la région Fc des anticorps. L'utilisation de ces molécules alternatives est donc forcément restreinte à des applications particulières non ou mal remplies par les anticorps entiers. On note toutefois une forte augmentation de ce type de molécules dans les essais cliniques de stade précoce et cette tendance augmente avec les années [19].

Nous décrivons ici les fragments d'anticorps approuvés. À ce jour, la seule charpente alternative aux anticorps ayant reçu une autorisation de mise sur le marché (AMM) est le Kalbitor ${ }^{\circledR}$, un inhibiteur de protéase dérivé des domaines Kunitz (domaines protéiques de 50-60 acides aminés inhibant l'activité des protéases), pour le traitement de l'angioœdème héréditaire [13]. Les développements cliniques sont poursuivis sur bien d'autres charpentes, avec certaines de ces molécules en phase III comme la DARPin ${ }^{\circledR}$ abicipar, dirigée contre le VEGF-A (vascular endothelial growth factor $A$ ).

\section{Le format Fab}

Le Fab, de par l'ancienneté de sa découverte, a été le premier fragment d'anticorps approuvé en clinique. L'abciximab est un Fab chimérique approuvé en 1993 et utilisé comme antiagrégant plaquettaire. Ce Fab interagit avec une protéine de surface des plaquettes empêchant ainsi la fixation de facteurs de coagulation comme le fibrinogène ou le facteur Willebrand. Le certolizumab pégol est un Fab humanisé reconnaissant et inhibant la fixation du TNF $\alpha$ (tumor necrosis factor alpha) à ses récepteurs, approuvé en 2009 dans le traitement de la polyarthrite rhumatoïde. Cette molécule présente la particularité d'être conjuguée à une molécule de polyéthylène glycol, ce qui augmente de façon importante sa demi-vie plasmatique à 14 jours et permet l'espacement des injections. L'idarucizumab est un fragment Fab humanisé antidote du dabigatran (Pradaxa ${ }^{\circledR}$ / Praxbind ${ }^{\circledR}$ ), une molécule anticoagulante inhibant la thrombine. Cet antidote peut être utilisé pour inverser rapidement et spécifiquement l'effet de cet anticoagulant en cas d'hémorragie grave ou de besoin d'intervention chirurgicale en urgence. Finalement, le ranibizumab est un fragment Fab humanisé ciblant le VEGF-A et approuvé en 2006 dans le traitement de certaines formes de la dégénérescence maculaire liée à l’âge (DMLA).

\section{Le format Fv}

Un des intérêts du format simple-chaîne des scFv est qu'il permet la construction facile de molécules multifonctionnelles. C'est cette propriété qui a été exploitée pour la première molécule approuvée utilisant ce format. Le blinatumomab est un dimère de scFv en tandem, le premier scFv reconnaissant un antigène exprimé par la cellule tumorale, CD19, le second une protéine de surface des lymphocytes $T$, la molécule $\operatorname{CD} 3 \varepsilon$, l'une des sous-unités du TcR ( $T$-cell receptor). De par sa bi-spécificité, le blinatumomab induit la formation d'une synapse cytolytique entre le lymphocyte $T$ et la cellule tumorale, où des enzymes protéolytiques sont libérées pour tuer les cellules cibles et ceci indépendamment de la spécificité du récepteur du lymphocyte T. Le blinatumomab a obtenu une AMM en 2014 pour le traitement de la leucémie aiguë lymphoblastique à précurseurs $B(L A L-B)$. De par sa très courte demi-vie plasmatique, cette molécule doit être administrée en perfusion continue. Un nouveau traitement à base de Fv a été approuvé en 2018, le moxétumomab pasudotox, dans le traitement des leucémies à tricholeucocytes. Cette molécule est une immunotoxine obtenue par la fusion d'un Fv stabilisé par un pont disulfure interchaîne, reconnaissant CD22, à l'exotoxine de Pseudomonas aeruginosa. La partie Fv permet le ciblage spécifique de cette toxine hautement active sur les cellules malignes et leur destruction par l'action de cette dernière qui pénètre dans la cellule et inhibe la synthèse des protéines.

\section{Le format VHH}

Plusieurs molécules fondées sur des répétitions de domaines VHH de camélidés sont en développement, ces domaines pouvant servir à la reconnaissance de la cible ou à l'augmentation de la demi-vie plasmatique par liaison à l'albumine. $\varepsilon$ n 2018, une première molécule, le caplacizumab a obtenu son agrément pour le traitement d'un désordre hémolytique d'origine autoimmune, le purpura thrombotique thrombocytopénique acquis. Le caplacizumab est un tandem du même VHH dirigé contre le facteur Willebrand et bloque l'agrégation plaquettaire. Cette nouvelle molécule a montré peu d'effets toxiques et une remarquable efficacité dans cette maladie rare mais présentant une mortalité élevée à court terme chez les patients. Elle représente très certainement le prototype de toute une famille de molécules fondées sur ces nouveaux formats d'anticorps.

\section{Un peu de prospective}

Avec le développement intensif de multiples anticorps thérapeutiques, la compréhension des mécanismes physiopathologiques évolue et se précise, généralement par les informations recueillies lors d'essais cliniques 
aboutissant à une faible efficacité, voire à un échec. Deux voies d'amélioration sont plus particulièrement étudiées: I'identification de nouvelles cibles et la combinaison des mécanismes d'action. Ces deux approches mettent en évidence le besoin aigu de méthodes et formats permettant l'association et l'assemblage rapide et flexible de plusieurs spécificités.

Certaines familles de protéines, comme les canaux ioniques ou les récepteurs couplés aux protéines $G(R C P G)$, sont depuis longtemps les cibles privilégiées des petites molécules mais leur ciblage avec des anticorps a été peu exploré. En effet, il ne s'agit pas uniquement de cibler ces récepteurs, mais également d'induire des effets pharmacologiques. Bien qu'il soit possible d'utiliser des anticorps entiers pour ces applications, le mode d'action quasi chirurgical désiré rend les petits formats particulièrement attractifs. Par exemple, de nombreuses molécules ciblant les RCPG avec des activités de modulation originales ont été décrites, notamment fondées sur des $\mathrm{VHH}$ de camélidés. Il est certain que ce champ va rapidement s'étendre dans les prochaines années avec de nombreux fragments d'anticorps et de charpentes à activité pharmacologique.

La réponse aux efficacités faibles des monothérapies consiste en la co-administration de plusieurs agents à la coopérativité espérée, notamment par le ciblage concomitant de plusieurs voies. C'est ainsi que de nombreux anticorps thérapeutiques à visée anti-tumorale sont actuellement revisités dans le cadre d'essais cliniques en association avec des inhibiteurs de points de contrôle immunitaire (anti-PD-1 [programmed cell death-1] ou anti-PD-L1 [programmed cell deathligand 1]) ou d'inhibiteur d'angiogenèse (anti-VEGF-A) [20]. Les résultats actuels laissent toutefois penser qu'une meilleure activité s'accompagne souvent d'un renforcement des effets secondaires. À la question du ciblage simultané de plusieurs voies, s'ajoute celle de la spécificité d'adressage de ces combinaisons thérapeutiques en faveur des tissus malades par rapport aux tissus sains. L'environnement tumoral, par exemple, conduit à la surexpression associée de plusieurs biomarqueurs de surface donnant aux cellules à cibler un profil unique et différencié des cellules dans un environnement sain. En ciblant ce profil différencié plutôt qu'un marqueur individuel qui pourra aussi être retrouvé au niveau des tissus sains, il devient possible d'augmenter la sélectivité des traitements anti-cancéreux. Cela peut s'obtenir par le renversement d'un paradigme prégnant dans le développement des anticorps associant forte affinité à forte spécificité, en développant des pinces moléculaires ciblant plusieurs cibles simultanément. La spécificité de ces pinces moléculaires pour les tissus cancéreux viendra de leur capacité faible d'engagement en absence d'une de leur cible. Ainsi, on assiste aujourd'hui à la construction de molécules thérapeutiques visant à combiner plusieurs spécificités pour plus d'efficacité et/ou sélectivité. Une voie majeure de développement d'alternatives aux AcM consiste donc à former des assemblages multispécifiques, que ce soit entre ces nouveaux formats ou par couplage à des anticorps (voir par exemple les conjugués FynomAbs, Fynomersanticorps, CD33 \& CD3 [21]). L'assemblage en une seule protéine de fusion de plus de 4 formats actifs a été réalisé par plusieurs sociétés. Outre leur facilité d'assemblage, l'originalité de la conception de ces combinaisons repose sur l'ingénierie de la coopérativité des mécanismes d'action, sans chercher à associer les molécules les plus efficaces individuellement afin de limiter la toxicité en même temps qu'augmenter l'efficacité. C'est ce que rend possible l'ingénierie de ces nouveaux Lego ${ }^{\circledR}$ protéiques. Par ailleurs, l'association en une seule molécule réduit la complexité des développements et finalement le coût des traitements.

Enfin, de nouvelles technologies telles que les nanoparticules pour la délivrance de produits, les vecteurs viraux pour la thérapie génique ou les cellules $T$ porteuses de récepteurs chimériques (CAR-T) (chimeric antigen receptor $T$ cell) sont également porteuses de grands espoirs de traitement. L'adressage de ces objets est une problématique essentielle. Cette fonction nouvelle doit être apportée sans affecter leurs propriétés natives, et l'on conçoit bien que des vecteurs de très petite taille sont particulièrement attractifs pour les diriger vers les organes et cellules d'intérêt. Là encore, l'ingénierie appliquée à de petites protéines a démontré à l'échelle du laboratoire, mais également clinique avec I'homologation des CAR-T utilisant des ScFv pour leur adressage, la grande compatibilité des petits fragments ou des charpentes protéiques pour remplir ce rôle. Le développement pharmaceutique de ces néo-objets est encore balbutiant, essentiellement pour des raisons de complexité réglementaire, mais devrait à n'en pas douter constituer une voie importante pour la génération de nouvelles approches thérapeutiques. $\diamond$

\section{SUMMARY}

Antibody alternative formats: antibody fragments and new frameworks

Antibodies are now recognized as routine molecules in many therapeutic fields, no longer restricted to oncology and inflammation. This explosion of the field leads to new needs that can be better fulfilled by molecules inspired but different from conventional antibodies. In particular, the antibody molecule has multiple functions that are not always necessary, such as its ability to recruit immune system cells, its bivalency, or its high plasma half-life. However, in most applications, its remarkable ability to recognize almost any molecular partner with high affinity and specificity must be preserved. In addition, antibodies are very large molecules, expensive to produce and having limited physicochemical properties that limit their use in aggressive media. Finally, in certain therapeutic applications, the large size of the antibody molecule may also limit its diffusion in tissues and prevent the recognition of some poorly accessible molecular structures. To address these limitations, many alternative formats to whole antibodies 
have been developed over the last twenty years. These new formats have found applications in many fields like biotechnology, in vitro and in vivo diagnosis, and therapy. Two large families of molecules cover this field and will be presented in this mini-review. The first family is based on antibody by reducing its size, such as classical antibody fragments (Fab, scFv) or those derived from camels or sharks (VHH, V-NAR). The second family was developed by first identifying frameworks fulfilling the desired properties, in particular the stability in extreme medium and the productivity in simple and economic systems like bacteria, then by grafting binding properties comparable to antibodies using methods based on in vitro directed molecular evolution techniques. This mini-review will focus on the most advanced molecules but the field is quickly evolving. It should be noted that many of these molecules, or even these approaches, are covered by patents and are often developed by young innovative companies, some of which have been already bought by large pharmaceutical groups. $\diamond$

\section{LIENS D'INTÉREิT}

Les auteurs déclarent n'avoir aucun lien d'intérêt concernant les données publiées dans cet article.

\section{RÉFÉRENCES}

1. Ward ES, Güssow D, Griffiths AD, et al. Binding activities of a repertoire of single immunoglobulin variable domains secreted from Escherichia coli. Nature 1989 ; 341 : 544-6.

2. Hamers-Casterman C, Atarhouch T, Muyldermans S, et al. Naturally occurring antibodies devoid of light chains. Nature $1993 ; 363: 446-8$.

3. Dumoulin M, Conrath K, Van Meirhaeghe A, et al. Single-domain antibody fragments with high conformational stability. Protein Sci. Publ. Protein Soc $2002 ; 11$ : 500-15.

4. Greenberg AS, Avila D, Hughes $M$, et al. A new antigen receptor gene family that undergoes rearrangement and extensive somatic diversification in sharks. Nature $1995 ; 374: 168-73$.

5. Oliveira S, Dongen GAMS van, Stigter-van Walsum M, et al. Rapid visualization of human tumor xenografts through optical imaging with a near-infrared fluorescent anti-epidermal growth factor receptor nanobody. Mol Imaging 2012 ; 11 : 33-46.

6. Weis W, Brown JH, Cusack S, et al. Structure of the influenza virus haemagglutinin complexed with its receptor, sialic acid. Nature $1988 ; 333: 426-31$.
7. Stijlemans B, Conrath K, Cortez-Retamozo V, et al. Efficient targeting of conserved cryptic epitopes of infectious agents by single domain antibodies. African trypanosomes as paradigm. J Biol Chem $2004 ; 279$ : 1256-61.

8. Jemmerson R. Multiple overlapping epitopes in the three antigenic regions of horse cytochrome cl. J Immunol 1987 ; 138 : 213-9.

9. Newman MA, Mainhart CR, Mallett CP, et al. Patterns of antibody specificity during the BALB/c immune response to hen eggwhite lysozyme. J Immunol $1992 ; 149: 3260-72$

10. Robin G, Sato Y, Desplancq D, et al. Restricted diversity of antigen binding residues of antibodies revealed by computational alanine scanning of 227 antibody-antigen complexes. J Mol Biol $2014 ; 426$ : 3729-43.

11. Weidle UH, Auer J, Brinkmann U, et al. The emerging role of new protein scaffold-based agents for treatment of cancer. Cancer Genomics Proteomics 2013; $10: 155-68$.

12. Yu X, Yang YP, Dikici $\varepsilon$, et al. Beyond antibodies as binding partners: The role of antibody mimetics in bioanalysis. Annu Rev Anal Chem Palo Alto Calif $2017 ; 10: 293-320$.

13. Vazquez-Lombardi R, Phan TG, Zimmermann C, et al. Challenges and opportunities for non-antibody scaffold drugs. Drug Discov Today $2015 ; 20$ : 1271-83.

14. Hanes J, Schaffitzel C, Knappik A, et al. Picomolar affinity antibodies from a fully synthetic naive library selected and evolved by ribosome display. Nat Biotechnol $2000 ; 18$ : 1287-92.

15. Orcutt KD, Adams GP, Wu AM, et al. Molecular simulation of receptor occupancy and tumor penetration of an antibody and smaller scaffolds: application to molecular imaging. Mol Imaging Biol 2017 ; 19 : 656-64.

16. Goux M, Becker G, Gorré $H$, et al. Nanofitin as a new molecular-imaging agent for the diagnosis of epidermal growth factor receptor over-expressing tumors. Bioconjug Chem $2017 ; 28: 2361-71$.

17. Schmidt MM, Wittrup KD. A modeling analysis of the effects of molecular size and binding affinity on tumor targeting. Mol Cancer Ther $2009 ; 8: 2861-71$.

18. Wittrup KD, Thurber GM, Schmidt MM, et al. Practical theoretic guidance for the design of tumor-targeting agents. Methods Enzymol $2012 ; 503$ : 255-68.

19. Nelson AL, Reichert JM. Development trends for therapeutic antibody fragments. Nat Biotechnol $2009 ; 27$ : 331-7.

20. Henricks LM, Schellens JHM, Huitema ADR, et al. The use of combinations of monoclonal antibodies in clinical oncology. Cancer Treat Rev 2015; $41: 859-67$.

21. Klupsch K, Baeriswyl V, Scholz R, et al. COVA4231, a potent CD3/CD33 bispecific FynomAb with IgG-like pharmacokinetics for the treatment of acute myeloid leukemia. Leukemia 2019 ; 33(3) : 805-8.

TIRÉS À PART

P. Martineau

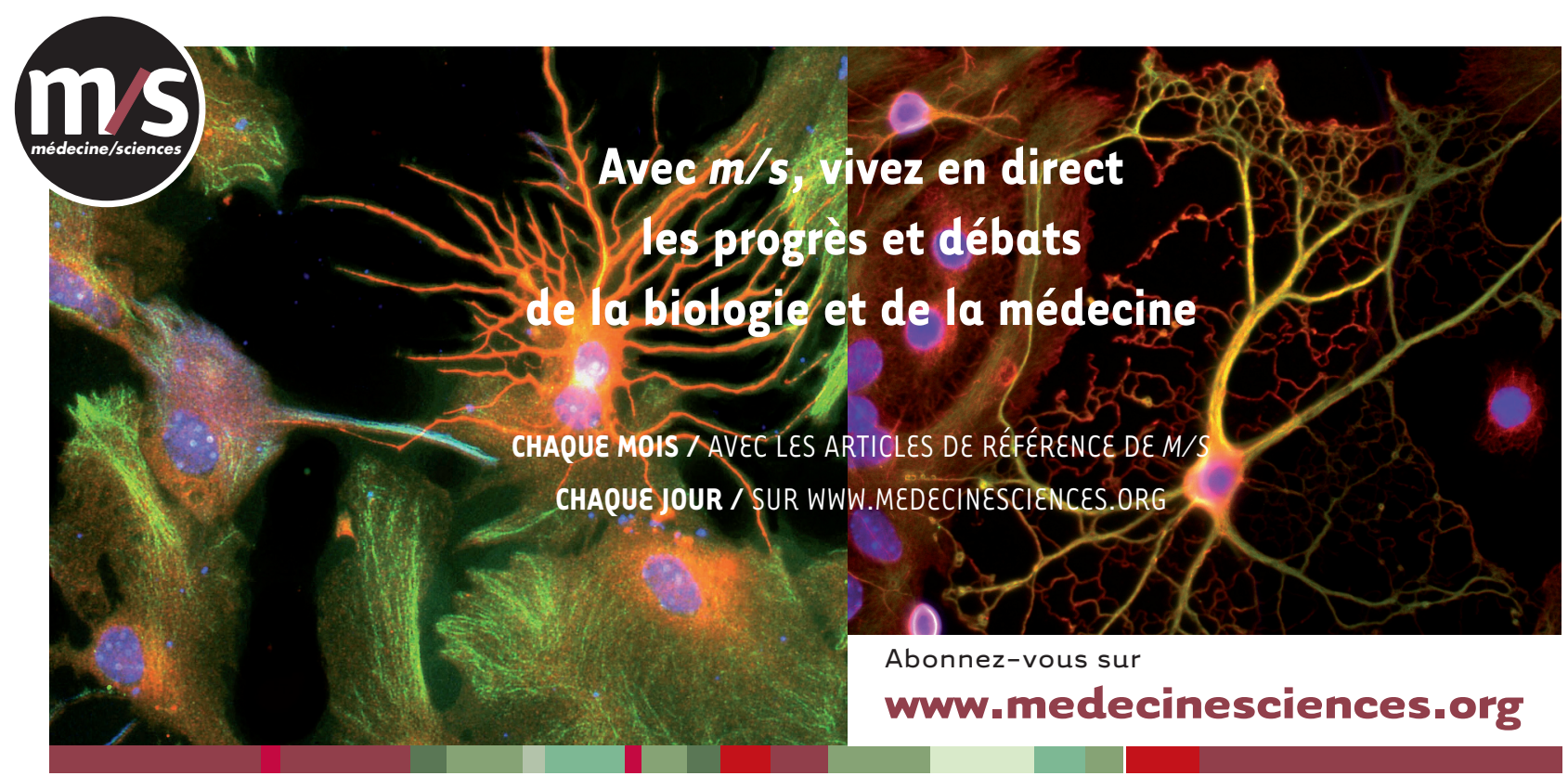

